\title{
Combined preoperative traction with instrumented posterior occipitocervical fusion for severe ventral brainstem compression secondary to displaced os odontoideum: technical report of 2 cases
}

\author{
Muhammad M. Abd-El-Barr, MD, PhD, ${ }^{1}$ Brian D. Snyder, MD, PhD, ${ }^{2}$ John B. Emans, MD, ${ }^{2}$ \\ Mark R. Proctor, MD, ${ }^{1}$ and Daniel Hedequist, MD² \\ Departments of ${ }^{1}$ Neurosurgery and ${ }^{2}$ Orthopedic Surgery, Boston Children's Hospital, Harvard Medical School, Boston, \\ Massachusetts
}

\begin{abstract}
Severe os odontoideum causing ventral brainstem compression is a rare and difficult entity to treat. It is generally accepted that severe os odontoideum causing ventral brainstem compression and neurological deficits warrants surgical treatment. This often requires both anterior and posterior procedures. Anterior approaches to the craniocervical junction are fraught with complications, including infection and risk of injury to neurovascular structures. External traction systems traditionally require long-term bedrest.
\end{abstract}

The authors report 2 cases of severe ventral brainstem compression secondary to displaced os odontoideum and describe their use of extended preoperative halo vest traction to reduce the severe kyphosis and improve neurological function, followed by posterior occipitocervical fusion. Postoperatively both patients showed remarkable improvements in their neurological function and kyphotic deformity.

Preoperative halo vest traction combined with posterior occipitocervical fusion appears to be a safe and effective method to treat brainstem compression by severe os odontoideum. It allows for adequate decompression of ventral neural structures and improvement of neurological function, but it is not hindered by the risks of anterior surgical approaches and does not restrict patients to strict bedrest as traditional traction systems. This method of halo vest traction and posterior-only approaches may be transferable to other cervical instability issues with both anterior and posterior pathologies.

http://thejns.org/doi/abs/10.3171/2016.5.PEDS16122

KEY WORDS posterior occipitocervical fusion; spine; brainstem compression; traction; os odontoideum

$\mathrm{O}$ $\mathrm{s}$ odontoideum is a general term describing a lack of continuity between the odontoid process and the body of C-2 (axis) and, more specifically, an independent ossicle with smooth cortical margins separated from a shortened odontoid peg. ${ }^{1}$ Although initially described by Giacomini in 1886, much of the etiology and optimal management is still under debate. The condition derives its name from the Latin forms for bone (os) and tooth (odontoideum). ${ }^{1}$ There are two main anatomical types described-orthotopic and dystopic. ${ }^{5,24}$ The orthotopic type describes an ossicle that is related to the anterior arch of C-1 (atlas) and can thus be reduced, while the dystopic describes the situation in which the ossicle is fused to the basion.
There is no consensus about the optimal treatment of os odontoideum, but it is recognized that patients with evidence of ventral craniocervical compression and neurological deficits require surgical decompression and stabilization. ${ }^{1,3,22}$ Numerous methods have been describedanterior approaches, posterior approaches, and a combination of both. ${ }^{1,3,8,10,11,22,25}$ Traction is also an important adjunct in the management of these rare entities, as instability plays an important role in the manifestation of symptoms for os odontoideum. ${ }^{17,21}$ Traditionally, traction is done with Gardner-Wells tongs, which leaves the patients immobilized. ${ }^{17}$ Others have described tightening the bolts on the halo crown to cause progressive distraction. ${ }^{12,21}$ For children, this may be difficult to maintain for a prolonged 
period of time. We have recently implemented a system by which patients are fitted with a halo vest and, over an extended period of time, more weight is added to gravity traction (Fig. 1). Although we have had success using acute traction and stabilization, ${ }^{10}$ it is our feeling that more prolonged amounts of time with traction allow for greater reduction of the basilar invagination. During treatment, the patients are allowed to ambulate with a specialized traction walker and use a wheelchair attached to traction, which allows mobilization throughout the hospital, affording time for daily activities. The traction may be applied to just the halo crown, but with more severe deformities, we attach the halo vest with nonrigid fixation to the crown to allow for longitudinal traction while at the same time providing protection from sudden rotation or bending. Patients' neurological function is continually assessed as more weight is added, and intermittent radiographs are obtained to monitor correction of the displaced os odontoideum and correction of the kyphotic deformity. It is our practice to add approximately $1 \mathrm{~kg} /$ day with continuous monitoring of the neurological examination and weekly radiographs to assess for improvement in the kyphotic deformity associated with the os odontoideum. Traction is applied 24 hours a day with the exception of nursing care such as showering.

In this paper, we review 2 cases of severely displaced os odontoideum with associated ventral brainstem compression and our experience with halo vest gravity traction and posterior-only instrumented fusion, with an emphasis on safety, reduction of deformity, and neurological outcome.

\section{Case Reports Case 1}

The patient was a 12-year-old girl weighing $30 \mathrm{~kg}$ from Nepal; she had trisomy 21 and a history of a traumatic fall when she was 6 years old. Immediately afterward, she was ambulatory, without any significant deficits. However, over the ensuing month, she developed worsening left-sided weakness (upper and lower extremity). This resulted in worsening ability to ambulate and notable verbal declines. Prior to the fall, the patient was conversational, able to ambulate without assistance including running, was potty trained, and social with multiple friends. She was unable to receive surgery in her native country and subsequently presented to another institution with radiographic evidence of severely displaced os odontoideum, evidence of spinal cord edema, and spinal cord intrinsic signal change. She underwent an occiput-C4 fusion at the age of 8, but, shortly after surgery, the occipital fixation failed. She experienced some improvement in her ambulatory and cognitive functions after this initial surgery, but the instrumentation needed to be removed approximately 10 months after her initial surgery due to implant failure and infection.

After instrumentation removal, the child's condition deteriorated with progressive weakness and worsening ability to ambulate. She also had motor dysfunction, primarily on the left side but also weakness in the right lower extremity. Additionally, the family reported fecal and urinary incontinence over the 3 months following implant removal. At presentation to our institution, she

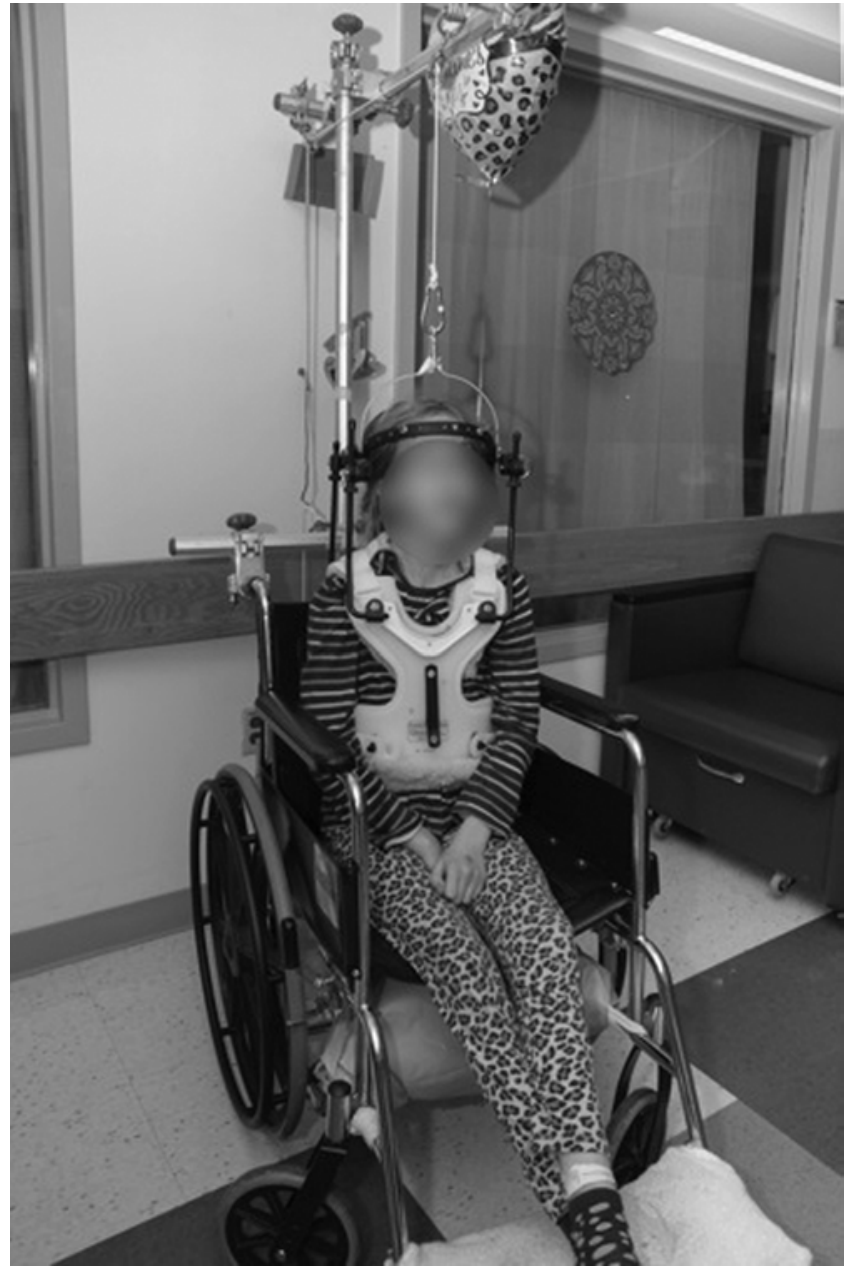

FIG. 1. Photograph of the patient in the halo vest traction system. During treatment the patients are allowed to ambulate with a specialized traction walker and to use a wheelchair attached to traction that allows mobilization throughout the hospital, affording time for daily activities. Traction may be applied to just the halo crown; however, with more severe deformities, we attach the halo vest with nonrigid fixation to the crown to allow for longitudinal traction while at the same time providing protection from sudden rotation or bending.

was severely myelopathic and could not ambulate. CT of the cervical spine revealed progressive deformity with severely displaced os odontoideum (Fig. 2 left). Repeat MRI demonstrated anterior and inferior displacement of the cranium-C1-os complex with severe cord compression and spinal cord signal abnormality (Fig. 2 right). The degree of deformity was thought to be unacceptable for an in situ fusion and the plan was made for gradual reduction of the invagination with halo vest gravity traction. Given her severe myelopathy along with her cognitive function, it was believed that placing her in a halo vest attached to the crown would prevent any significant bending/rotation in traction that would further compromise her remaining cord function.

The patient was taken to the operating room for halo vest fixation, with 10 pins each tightened to 5 inch-pounds, and traction was applied for 4 weeks with a maximum weight of 18 pounds. Following this period of traction, the child had 

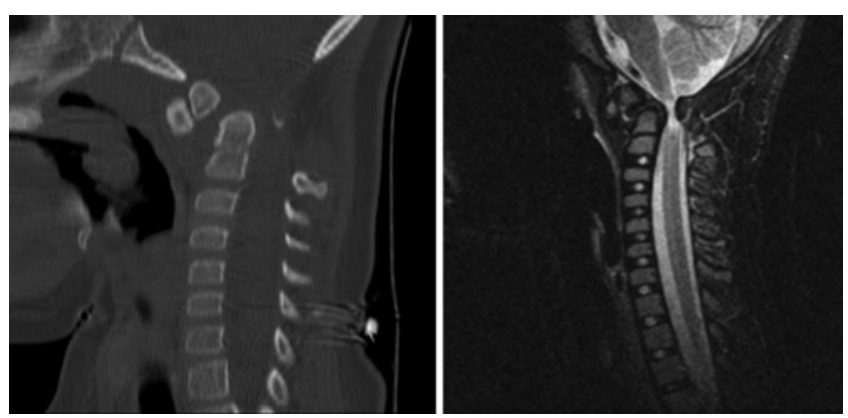

FIG. 2. Case 1. Left: Sagittal CT scan showing severely displaced os odontoideum. Right: T2-weighted MR image demonstrating severe ventral brainstem compression with evidence of signal change in the spinal cord. The patient underwent occiput-C4 posterior lateral mass screw and rod fixation and fusion at another institution. Occipital fixation failed due to screw pullout and the patient ultimately required removal of the posterior instrumentation.

considerable improvement in her cervical deformity (Fig. 3C). After spinal realignment was achieved, she was then taken to the operating room to undergo decompression of the foramen magnum and occiput-C5 fusion. It was noted that the vertebral artery had a tortuous path on the left, so at C-2 only a right-sided pedicle screw was placed. Lateral mass screws were placed bilaterally from C-3 to C-5. Iliac crest bone was harvested for autograft, and recombinant human bone morphogenetic protein (Medtronic) was used for allograft.
Correction of the ventral brainstem compression was maintained immediately postoperatively (Fig. 3D). The child had improvements in her neurological function, becoming once again able to ambulate on her own, and this improvement was maintained at the 2-year follow-up. Lateral radiography revealed maintenance of good alignment and fusion (Fig. 3E).

\section{Case 2}

The patient was a 14-year-old girl weighing $50 \mathrm{~kg}$ from the Dominican Republic, who had normal development and no history of trauma but was noted to be having increasing problems with ambulation. MRI at that time revealed severely displaced os odontoideum with ventral brainstem compression and evidence of signal change in the spinal cord (Fig. 4A). Unfortunately, due to international visa issues, it took approximately 4 months before she arrived at our institution. By that time, her condition had deteriorated significantly and she was no longer ambulatory, had developed significant myelopathy, and urinary and fecal incontinence. CT scanning revealed a severely displaced os odontoideum and severe kyphotic deformity associated with severe ventral brainstem compression (Fig. 4B).

The patient underwent halo ring fixation, with pins tightened to 8 inch-pounds, and traction was applied for 4 weeks with a maximum weight of 25 pounds. The vest was used in a nonrigid fashion to allow for further distraction but no significant movement in the sagittal or coronal plane. The patient had marked improvement in her defor-
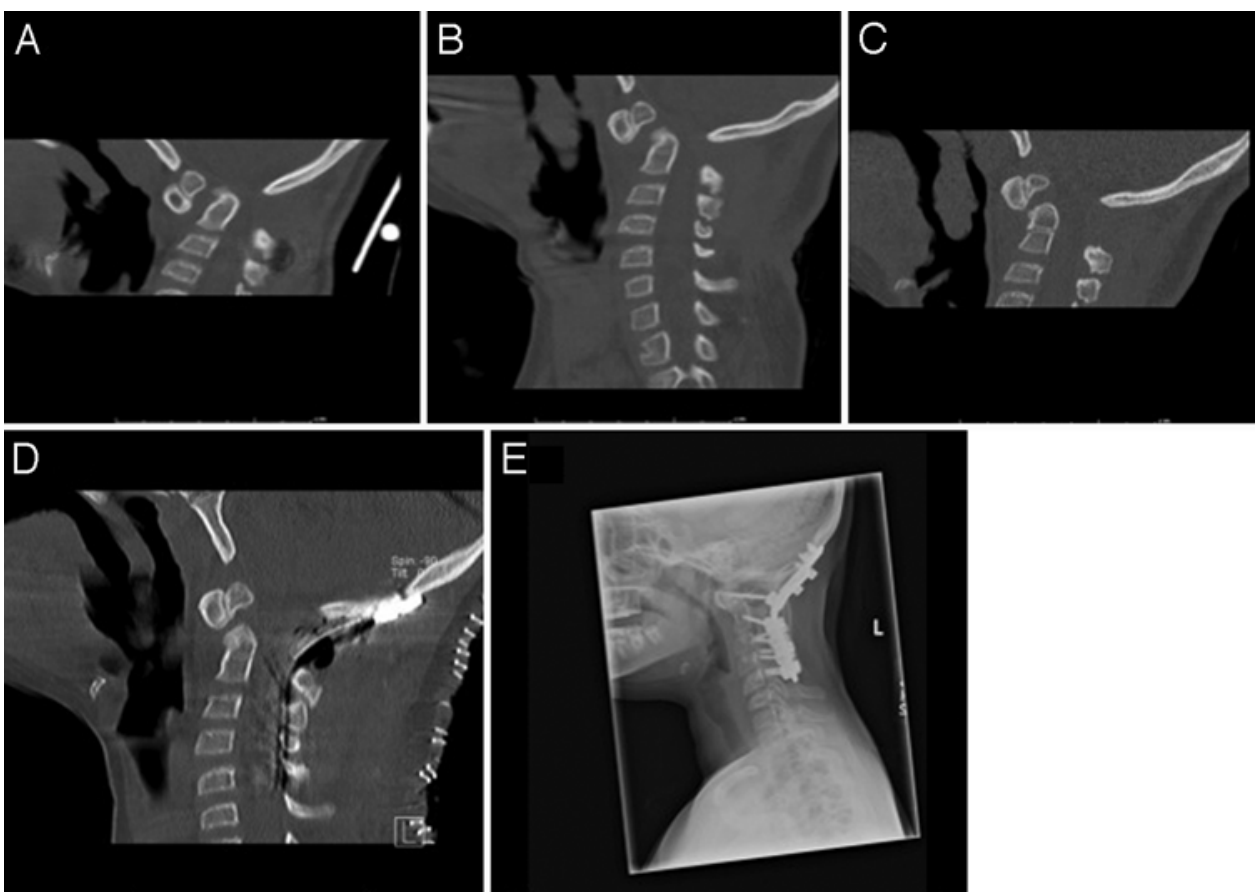

FIG. 3. Case 1. A: Sagittal CT scan revealing continued severely displaced os odontoideum and ventral brainstem compression. B: After 10 days and 13 pounds of traction, there is improvement in the kyphotic deformity and the ventral brainstem compression. C: After 28 days of traction with a maximum weight of 18 pounds, a sagittal CT scan shows improvement of angle of os odontoideum and increase in the spinal cord diameter. D: Immediately postoperatively, a CT scan demonstrates continued improvement in the kyphotic deformity. E: Lateral radiograph 2 years postoperatively revealing stable angulation of os odontoideum. 

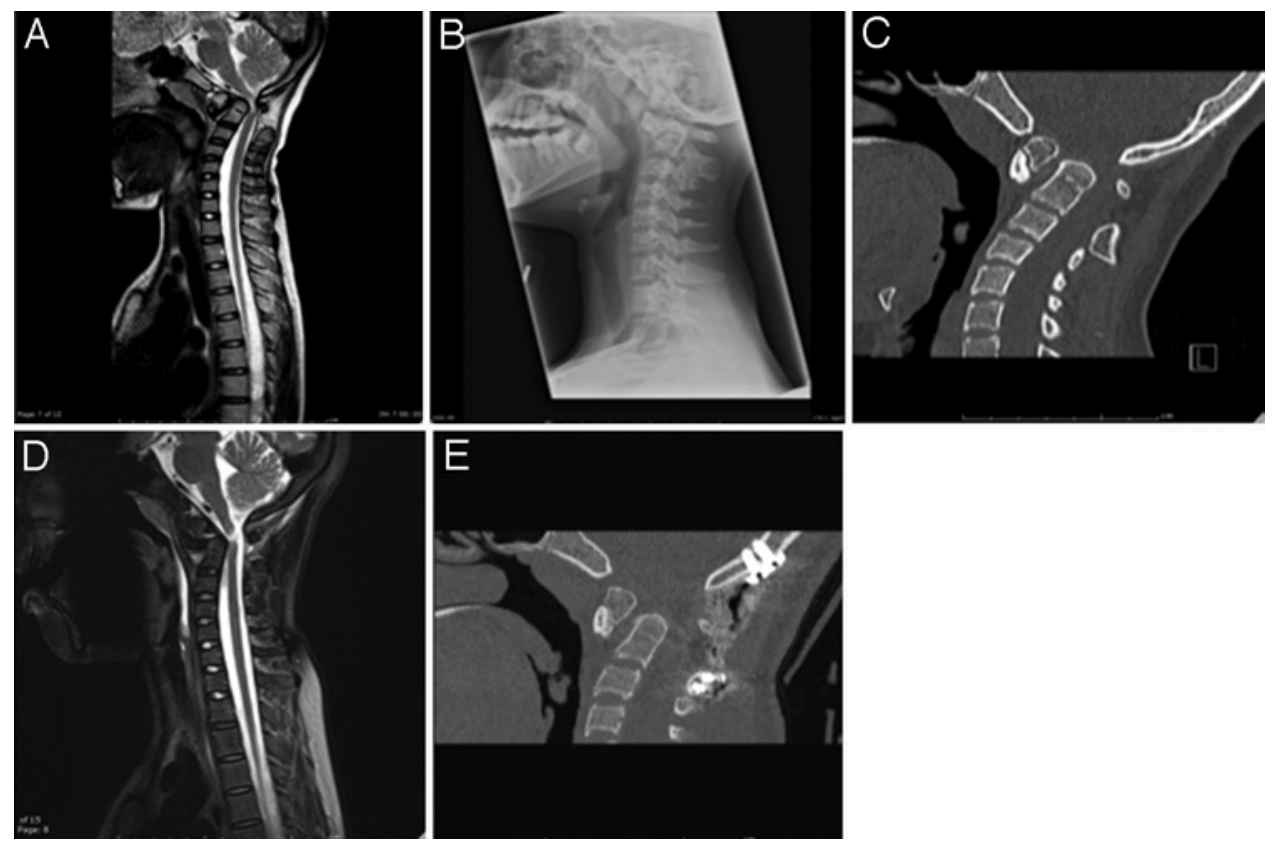

FIG. 4. Case 2. A: Sagittal T2-weighted MR image revealing significant kyphotic deformity and evidence of cord signal change. B: Six months later, the patient had become incontinent and nonambulatory. A lateral radiograph reveals worsening kyphotic deformity. C: After 10 days and 17 pounds of traction, a sagittal CT scan shows gradual improvement in kyphotic deformity. D: After long-term traction (28 days) with a maximum weight of 25 pounds, the patient had improvement in incontinence. Sagittal MR image reveals improvement in kyphotic deformity and increased caliber of spinal cord at cervicomedullary junction. E: Postoperatively, the patient continues to have improvements in her neurological status, and CT reveals marked improvement in kyphotic deformity.

mity, with MRI documenting great improvement in the space available for the cord (Fig. 4D). The patient the underwent occiput-C2 fusion with an occipital plate, C-1 lateral mass screws, and C-2 translaminar screws. Iliac crest bone was harvested for autograft, and recombinant human bone morphogenetic protein was used for allograft. Postoperatively, the patient continued to have improvements in her neurological status, and CT revealed marked improvement in kyphotic deformity (Fig. 4E). At short-term follow-up, she had regained bowel and bladder control and could ambulate independently assisted only by ankle foot orthoses.

\section{Discussion}

Os odontoideum can be a difficult entity to treat when associated with long-term displacement, brainstem compression, and myelopathy. There is no consensus regarding the etiology of this finding or the optimal treatment. For patients who are asymptomatic, some have advocated clinical and radiological surveillance, while others have advocated surgical intervention, especially if there is evidence of C1-2 instability. There are some anecdotal reports of spinal cord injury with minor trauma, ${ }^{22}$ reinforcing the possible benefit of stabilization in asymptomatic patients. For patients who present with neurological deficits, however, surgical intervention is warranted..$^{11,26}$ It is important to note that os odontoideum may be a solitary finding or associated with other findings of the craniocervical junction, including fractures of the posterior osseous ring or C-1 lateral mass. In a large series of cases of patients with os odontoideum who underwent surgery, 35 (12.5\%) of 279 had evidence of such associations. ${ }^{26}$ It is important to note that in the 2 cases presented there was no have evidence of these associations. However, it may be that, as long as these associations are not a contraindication for traction (e.g., an associated herniated cervical disc), the methods above can be used in conjunction with other posterior techniques to correct the associated findings.

Brainstem compression by the ossicle is perhaps the most extreme presentation of os odontoideum..$^{23}$ As with other etiologies of ventral brainstem compression, preoperative traction is often necessary. ${ }^{7,17,20}$ Posterior fusion alone is often unsuccessful, as seen in Case 1. Although we have good experience with intraoperative reduction, ${ }^{10}$ the kyphotic deformity in the present cases was too severe for this single-stage correction. As our experience increases with these cases, it may be that we are able to more accurately determine which patients will need intraoperative reduction and which will need this prolonged traction. In most cases, traction involves the use of Gardner-Wells tongs and gravity traction. This has the advantage of allowing for gradual increases in weight but has the distinct disadvantage of requiring patients to be confined to bed, although there are reports of mobile Gardner-Wells tongs system. ${ }^{17}$ There is also evidence that reusable GardnerWells tongs and MRI-compatible ones may have decreased pullout strength, and, as such, may put patients at risk for morbid complications. ${ }^{2,13}$ By applying a halo vest and gravity traction, we allow the patients to have more mobility and more comfort, while capturing the advantages of traction. Others have used similar halo vest gravity traction 
systems for the treatment of severe scoliosis. ${ }^{6,16,19}$ With regard to the amount of weight and how long the traction is applied, it has been our experience that gradual correction of the kyphotic deformity is better tolerated by the patients and may avoid complications of overcorrection. ${ }^{16}$ We have previously described our method of single-stage reduction and stabilization of basilar invagination after failed prior fusion surgery in children with Down syndrome..$^{10}$ In these cases, the kyphotic deformity was not as great and thus a more acute correction was thought to be more appropriate. It is also important to note that in both of the present cases, the os odontoideum was of the orthotopic type, where the ossicle is related to the anterior arch of $\mathrm{C}-1$, and not of the dystopic type, where the ossicle is fused to the basion. In those situations, a more direct correction of the ventral brainstem compression would be more appropriate.

By improving the kyphotic deformity and decreasing the ventral compression on the brainstem, we have the opportunity to perform a stabilization procedure posteriorly, relieving the patients from the myriad risks associated with anterior approaches, including infection, anterior neck swelling, and the morbidity of transoral and/or transnasal procedures. ${ }^{3,15}$ As outlined in an excellent review on the evolution of transoral and transnasal approaches to the craniovertebral junction, Dlouhy et al. suggested the use of acute cervical traction and the addition of intraoperative neuromuscular blockade and, if the pathology is still irreducible, a transnasal or transoral approach depending on if the pathology is above or below the hard palate. ${ }^{4}$ There is some evidence that, compared with transoral routes, transnasal approaches may have fewer complication risks, including decreased risks of infection, and could permit early extubation and early feeding. ${ }^{9,14,18}$ Our Case 1 suggests that a combined approach works better than a single approach, as a posterior decompression, fixation, and stabilization procedure had previously failed. Longer followup and more patients will be needed to address important issues such as fusion rates, but the positive results we achieved are a cause for optimism in these extremely difficult cases of brainstem compression and neurological compromise due to severely displaced os odontoideum. The fusion rate for most pediatric patients with modern instrumentation is high, and while the follow-up in our patients is limited, clearly they had a significant improvement in alignment and neurological status.

\section{Conclusions}

Brainstem compression due to severely displaced os odontoideum is a difficult entity to treat. It is clear that there is a need for both ventral decompression and posterior stabilization. By using long-term traction with a halo vest to decompress the anterior brainstem, we were able to avoid traditional transnasal and/or transoral approaches yet not confine the patient to long-term bedrest. Once the ventral compression is improved, a posterior-only approach appears adequate to maintain improved alignment. This method of halo vest traction and posterior-only approaches may be transferable to other cervical instability issues in cases involving both anterior and posterior pathologies.

\section{References}

1. Arvin B, Fournier-Gosselin MP, Fehlings MG: Os odontoideum: etiology and surgical management. Neurosurgery 66 (3 Suppl):22-31, 2010

2. Blumberg KD, Catalano JB, Cotler JM, Balderston RA: The pullout strength of titanium alloy MRI-compatible and stainless steel MRI-incompatible Gardner-Wells tongs. Spine (Phila Pa 1976) 18:1895-1896, 1993

3. Chaudhry NS, Ozpinar A, Bi WL, Chavakula V, Chi JH, Dunn IF: Basilar invagination: case report and literature review. World Neurosurg 83:1180.e7-1180.e11, 2015

4. Dlouhy BJ, Dahdaleh NS, Menezes AH: Evolution of transoral approaches, endoscopic endonasal approaches, and reduction strategies for treatment of craniovertebral junction pathology: a treatment algorithm update. Neurosurg Focus 38(4):E8, 2015

5. Fielding JW, Hensinger RN, Hawkins RJ: Os odontoideum. J Bone Joint Surg Am 62:376-383, 1980

6. Garabekyan T, Hosseinzadeh P, Iwinski HJ, Muchow RD, Talwalkar VR, Walker J, et al: The results of preoperative halo-gravity traction in children with severe spinal deformity. J Pediatr Orthop B 23:1-5, 2014

7. Goel A, Bhatjiwale M, Desai K: Basilar invagination: a study based on 190 surgically treated patients. J Neurosurg 88: 962-968, 1998

8. Goel A, Shah A: Atlantoaxial joint distraction as a treatment for basilar invagination: a report of an experience with 11 cases. Neurol India 56:144-150, 2008

9. Goldschlager T, Härtl R, Greenfield JP, Anand VK, Schwartz TH: The endoscopic endonasal approach to the odontoid and its impact on early extubation and feeding. J Neurosurg 122:511-518, 2015

10. Hedequist D, Bekelis K, Emans J, Proctor MR: Single stage reduction and stabilization of basilar invagination after failed prior fusion surgery in children with Down's syndrome. Spine (Phila Pa 1976) 35:E128-E133, 2010

11. Klimo P Jr, Coon V, Brockmeyer D: Incidental os odontoideum: current management strategies. Neurosurg Focus 31(6):E10, 2011

12. Kyoshima K, Kakizawa Y, Tokushige K: Simple cervical spine traction using a halo vest apparatus: technical note. Surg Neurol 59:518-521, 2003

13. Lerman JA, Haynes RJ, Koeneman EJ, Koeneman JB, Wong WB: A biomechanical comparison of Gardner-Wells tongs and halo device used for cervical spine traction. Spine (Phila Pa 1976) 19:2403-2406, 1994

14. Liu JK, Patel J, Goldstein IM, Eloy JA: Endoscopic endonasal transclival transodontoid approach for ventral decompression of the craniovertebral junction: operative technique and nuances. Neurosurg Focus 38(4):E17, 2015

15. Liu X, Wang H, Zhou Z, Jin A: Anterior decompression and fusion versus posterior laminoplasty for multilevel cervical compressive myelopathy. Orthopedics 37:e117-e122, 2014

16. Park DK, Braaksma B, Hammerberg KW, Sturm P: The efficacy of preoperative halo-gravity traction in pediatric spinal deformity the effect of traction duration. J Spinal Disord Tech 26:146-154, 2013

17. Peng X, Chen L, Wan Y, Zou X: Treatment of primary basilar invagination by cervical traction and posterior instrumented reduction together with occipitocervical fusion. Spine (Phila Pa 1976) 36:1528-1531, 2011

18. Ponce-Gómez JA, Ortega-Porcayo LA, Soriano-Barón HE, Sotomayor-González A, Arriada-Mendicoa N, GómezAmador JL, et al: Evolution from microscopic transoral to endoscopic endonasal odontoidectomy. Neurosurg Focus 37(4):E15, 2014

19. Rinella A, Lenke L, Whitaker C, Kim Y, Park SS, Peelle $\mathrm{M}$, et al: Perioperative halo-gravity traction in the treatment of severe scoliosis and kyphosis. Spine (Phila Pa 
1976) 30:475-482, 2005 [Erratum in Spine (Phila Pa 1976) 30:994, 2005]

20. Sawin PD, Menezes AH: Basilar invagination in osteogenesis imperfecta and related osteochondrodysplasias: medical and surgical management. J Neurosurg 86:950-960, 1997

21. Simsek S, Yigitkanli K, Belen D, Bavbek M: Halo traction in basilar invagination: technical case report. Surg Neurol 66:311-314, 2006

22. Smith JS, Shaffrey CI, Abel MF, Menezes AH: Basilar invagination. Neurosurgery 66 (3 Suppl):39-47, 2010

23. Taggard DA, Menezes AH, Ryken TC: Treatment of Down syndrome-associated craniovertebral junction abnormalities. J Neurosurg 93 (2 Suppl):205-213, 2000

24. Vargas TM, Rybicki FJ, Ledbetter SM, MacKenzie JD: Atlantoaxial instability associated with an orthotopic os odontoideum: a multimodality imaging assessment. Emerg Radiol 11:223-225, 2005

25. Visocchi M, Fernandez E, Ciampini A, Di Rocco C: Reducible and irreducible os odontoideum in childhood treated with posterior wiring, instrumentation and fusion. Past or present? Acta Neurochir (Wien) 151:1265-1274, 2009

26. Zhao D, Wang S, Passias PG, Wang C: Craniocervical instability in the setting of os odontoideum: assessment of cause, presentation, and surgical outcomes in a series of 279 cases. Neurosurgery 76:514-521, 2015

\section{Disclosures}

Dr. Emans reports receiving royalties from and being a consultant for DePuy Synthes Spine; he also reports being a consultant for Medtronic Spine.

\section{Author Contributions}

Conception and design: all authors. Acquisition of data: AbdEl-Barr, Proctor. Analysis and interpretation of data: Hedequist, Abd-El-Barr, Snyder, Proctor. Drafting the article: Hedequist, Abd-El-Barr, Proctor. Critically revising the article: all authors. Reviewed submitted version of manuscript: Hedequist, Abd-ElBarr, Proctor.

\section{Correspondence}

Daniel Hedequist, Department of Orthopedic Surgery, Boston Children's Hospital, Harvard Medical School, 300 Longwood Ave., Boston, MA 02115. email: daniel.hedequist@childrens. harvard.edu. 